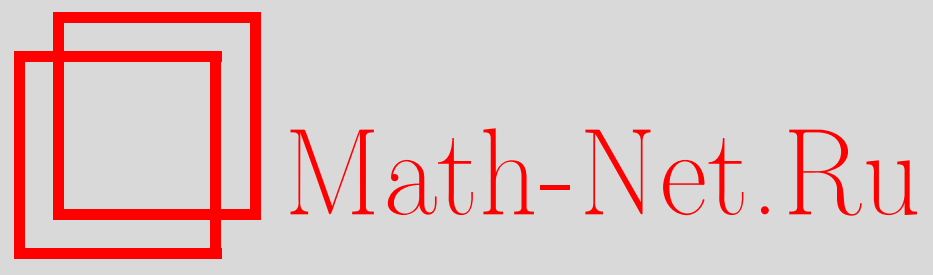

Д. Б. Рохлин, Задача о мартингальном выборе в случае конечного дискретного времени, Теория вероятн. и ее примен., 2005, том 50, выпуск 3, 480500

DOI: https://doi.org/10.4213/tvp90

Использование Общероссийского математического портала MathNet.Ru подразумевает, что вы прочитали и согласны с пользовательским соглашением

http://www.mathnet.ru/rus/agreement

Параметры загрузки:

IP : 3.81 .55 .215

26 апреля 2023 г., 18:19:26

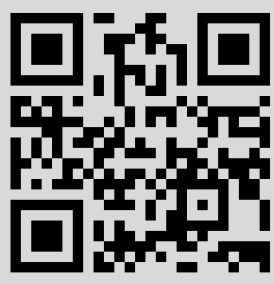




\section{ЗАДАЧА О МАРТИНГАЛЬНОМ ВЫБОРЕ В СЛУЧАЕ КОНЕЧНОГО ДИСКРЕТНОГО ВРЕМЕНИ}

Для многозначного случайного процесса с открытыми выпуклыми значениями, заданного на фильтрованном вероятностном пространстве, получен критерий существования согласованной с фильтрацией последовательности селекторов, которую можно презратить в мартингал путем эквивалентной замены меры. Данный критерий носит геометрический характер и выражен в терминах носителей регулярных условных верхних распределений элементов многозначного процесса.

Kлючевые слова и фразы: мартингальные меры, многозначные отображения, измеримый выбор, носители регулярных условных распределений, представление Кастэна.

Введение. Пусть на фильтрованном вероятностном пространстве $\left(\Omega, \mathscr{F},\left(\mathscr{F}_{n}\right)_{n=0}^{N}, \mathbf{P}\right), \mathscr{F}_{N}=\mathscr{F}$, задана последовательность $\mathscr{F}_{n}$-измеримых многозначных отображений $\omega \mapsto G_{n}(\omega) \subset \mathbf{R}^{d}, n=0,1, \ldots, N$, с непустыми открытыми выпуклыми значениями (определение измеримости многозначного отображения приводится ниже). Цель настоящей работы состоит в получении критерия существования следующей пары: согласованного с фильтрацией $\left(\mathscr{F}_{n}\right)_{n=0}^{N}$ однозначного $d$-мерного случайного процесса $\left(S_{n}\right)_{n=0}^{N}, S_{n}(\omega) \in G_{n}(\omega)$, и эквивалентной $\mathbf{P}$ вероятностной меры $\mathbf{Q}$, относительно которой $S$ является мартингалом. Если такая пара существует, то будем говорить, что разрешима задача о мартингальком вигборе.

Если, отказавшись от условия открытости, предположить, что отображения $G_{n}$ являются однозначными, то получим известную задачу о существовании эквивалентной мартингальной меры для заданного случайного процесса $S_{n}=G_{n}$. Эта проблема играет важную роль в финансовой математике, где предполагается, что процесс $S$ описывает динамику цен активов, и существование эквивалентной мартингальной меры является критерием безарбитражности рынка (см., например, [1]-[4]).

* Ростовский государственный университет, механико-математический факультет, ул. Зорге, 5, 344090 Ростов-на-Дону, Россия; e-mail: rokhlin@math.rsu.ru 
В работе [3] получен геометрический критерий существования эквивалентной мартингальной меры для $S$, состоящий в том, что выпуклые оболочки носителей регулярных условных распределений элементов $S_{n}-S_{n-1}$ относительно $\sigma$-алгебры $\mathscr{F}_{n-1}$ должны содержать начало координат в качестве относительно внутренней точки. Критерий настояшей работы в идейном плане является обобщением данного результата.

Заметим, что при наличии операционных издержек критерий безарбитражности состоит в наличии следующей пары: случайного процесса, расположенного между ценами покупки и продажи, и соответствующей эквивалентной мартингальной меры [5]. Далее, если $\lambda x \in G_{n}(\omega)$ для любых $\lambda>0, x \in G_{n}(\omega)$, то разрешимость задачи о мартингальном выборе эквивалентна существованию $\mathbf{P}$-мартингала $Y$, удовлетворяющего условию $Y_{n} \in G_{n}$. Действительно, если пара $S, \mathrm{Q}$ является решением задачи о мартингальном выборе, то положим $Y_{n}=z_{n} S_{n}$, где $z_{n}$ - процесс плотности меры $\mathbf{Q}$ относительно $\mathbf{P}$ :

$$
z_{N}=\frac{d \mathbf{Q}}{d \mathbf{P}}, \quad z_{n-1}=\mathbf{E}_{\mathbf{P}}\left(z_{n} \mid \mathscr{F}_{n-1}\right), \quad 1 \leqslant n \leqslant N .
$$

Тогда по формуле Байеса [4, гл. V, § 3a, п. 3]

$$
\mathbf{E}_{\mathbf{P}}\left(Y_{n} \mid \mathscr{F}_{n-1}\right)=\mathbf{E}_{\mathbf{P}}\left(z_{n} S_{n} \mid \mathscr{F}_{n-1}\right)=z_{n-1} \mathbf{E}_{\mathbf{Q}}\left(S_{n} \mid \mathscr{F}_{n-1}\right)=z_{n-1} S_{n-1}=Y_{n-1}
$$

В рамках моделей операционных издержек, предложенных в работах [6]-[8], при соответствуюшем выборе множеств $G_{n}$ сушествование указанного $\mathbf{P}$-мартингала $Y$ является критерием безарбитражности рынка.

Эти результаты и служат основной мотивировкой данной работы. Однако дальнейшие рассуждения не связаны непосредственно с финансовой проблематикой.

В п. 1 приводится ряд сведений, необходимых для формулировки и доказательства основной теоремы, которая сформулирована в п. 2 (теорема 1). Следуюцие два пункта посвящены ее доказательству. Наконец, в п. 5 рассматривается случай конечного вероятностного пространства. При этом удается снять условие открытости множеств $G_{n}(\omega)$, а доказательство аналога основной теоремы становится элементарным.

Отметим также, что лемма 3 представляет некоторый самостоятельный интерес.

\section{1. Необходимые сведения из теории измеримых много-} значных отображений. Рассмотрим вероятностное пространство $(\Omega, \mathscr{F}, \mathbf{P})$ и некоторую $\sigma$-алгебру $\mathscr{H} \subset \mathscr{F}$. Далее предполагается, что все рассматриваемые $\sigma$-алгебры являются полными относительно меры $\mathbf{P}$ (т.е. содержат все подмножества своих множеств Р-нулевой меры). 
Многозначное отображение $F$, относящее каждому $\omega \in \Omega$ некоторое подмножество $F(\omega) \subset \mathbf{R}^{d}$, называется $\mathscr{H}$-измеримым, если $\{\omega: F(\omega) \cap$ $V \neq \varnothing\} \in \mathscr{H}$ для любого открытого множества $V \subset \mathbf{R}^{d}$. Отображение $F$ называется замкнутым (выпуклым, открытым), если множества $F(\omega)$ замкнуты (выпуклы, открыты). График и эффективное множество $F$ определяются следующим образом:

$$
\operatorname{gr} F=\{(\omega, x): x \in F(\omega)\}, \quad \operatorname{dom} F=\{\omega: F(\omega) \neq \varnothing\}
$$

Обозначим через cl, int, ri, conv замыкание, внутренность, относительную внутренность и выпуклую оболочку подмножества конечномерного пространства. Пусть $\mathscr{B}=\mathscr{B}\left(\mathbf{R}^{d}\right)-\sigma$-алгебра борелевских подмножеств $\mathbf{R}^{d}$.

Функция $\xi: \Omega \mapsto \mathbf{R}^{d}$ называется селектором $F$, если $\xi(\omega) \in F(\omega)$ для всех $\omega \in \operatorname{dom} F$. Множество $\mathscr{H} \mid \mathscr{B}$-измеримых селекторов $F$ обозначим через $\mathscr{S}(F, \mathscr{H})$. Следуя [9], для элементов множества $\mathscr{S}\left(\mathbf{R}^{d}, \mathscr{H}\right)$ будем использовать запись $\xi \in \mathscr{H} \mid \mathscr{B}$.

В приводимых ниже предложениях собраны некоторые свойства измеримых многозначных отображений, используемые в дальнейшем.

Предложение 1. Для $\mathscr{H}$-измеримости замкнутого многозначного отображения $F$ необходимо и достаточно, чтобы существовало такое счетное множество $\left\{\xi_{i}\right\}_{i=1}^{\infty}, \xi_{i} \in \mathscr{S}(F, \mathscr{H})$, что множества $\left\{\xi_{i}(\omega)\right\}_{i=1}^{\infty}$ плотньв в $F(\omega)$ при каждом $\omega \in \operatorname{dom} F$.

Доказательство предложения 1 имеется, например, в [10, теорема 5.6], [11, с. 331]. Семейство $\left\{\xi_{i}\right\}_{i=1}^{\infty}$ будем называть представлением Кастэна (C. Castaing) для $F$.

Предложение 2. Пусть gr $F \in \mathscr{H} \otimes \mathscr{B}, \operatorname{mozда~} \mathscr{S}(F, \mathscr{H}) \neq \varnothing$.

Данный результат также хорошо известен: см., например, [12, теорема 1], [13, с. 56].

Предложение 3. Справедливы следующие утверждения.

(а) Многозначное отображение $F$ является $\mathscr{H}$-измеримьм тогда и только тогда, когда $\mathscr{H}$-измеримо $\mathrm{cl} F$.

(b) Если многозначные отображения $F_{i}, i \in \mathbf{N}$, являются $\mathscr{H}$-измеримыли, то отображение $\bigcup_{i=1}^{\infty} F_{i}$ также $\mathscr{H}$-измеримо.

(c) Eсли gr $F \in \mathscr{H} \otimes B$, то $F-\mathscr{H}$-измеримо. Если $F-\mathscr{H}$-измеpuмо, mo $\operatorname{gr}(\operatorname{cl} F) \in \mathscr{H} \otimes \mathscr{B}$.

(d) Eсли $F$ - $\mathscr{H}$-измеримо, то отображение conv $F$ также является $\mathscr{H}$-измеримылм.

(е) Если $F-\mathscr{H}$-измеримо и вылукло, то $\operatorname{gr}(\operatorname{ri} F) \in \mathscr{H} \otimes \mathscr{B}$, $\operatorname{gr}(\operatorname{int} F) \in \mathscr{H} \otimes \mathscr{B}$.

(f) Если $F_{1}$ и $F_{2}$ - $\mathscr{H}$-измеримь и $F_{1}$ открьто и вылукло, то $\operatorname{gr}\left(F_{1} \cap F_{2}\right) \in \mathscr{H} \otimes \mathscr{B}$. 
Утверждения (a) и (b) очевидны, (c) содержится в [10] (теоремы 3.4, 3.3). Доказательство измеримости conv $F$, приведенное в $[14$, лемма 1], является небольшой модификацией рассуждений $[10$, теорема 9.1], где рассматривались замкнутые отображения. Первая часть утверждения (е) доказана в [14, лемма 1]. Вторая часть данного утверждения следует из представления int $F=\operatorname{cl} F \backslash \partial(\mathrm{cl} F)$, поскольку измеримость графика границы $\partial(\mathrm{cl} F)$ измеримого замкнутого отображения $\mathrm{cl} F$ вытекает из теоремы 4.6 (ii) работы [10]. Наконец, справедливость (f) является следствием равенства $F_{1} \cap F_{2}=F_{1} \cap\left(\mathrm{cl} F_{2}\right)$ и пп. (с) и (е).

Пусть $B_{\varepsilon}(x) \subset \mathbf{R}^{d}$ - открытый шар радиуса $\varepsilon$ с центром в точке $x \in \mathbf{R}^{d}$. Далее будет использоваться следующий известный результат (см. [15, с. 338]): отображение $\omega \mapsto B_{\delta(\omega)}(\xi(\omega)) \subset \mathbf{R}^{d}$ (измеримая трубка) является $\mathscr{H}$-измеримым, если $\delta$ - положительная $\mathscr{H}$-измеримая случайная величина и $\xi \in \mathscr{H} \mid \mathscr{B}$.

Если многозначное отображение $F$ замкнуто и $\operatorname{dom} G=\Omega$, то можно считать, что $F$ является однозначным отображением $\Omega$ в множество $\mathrm{CL}=\mathrm{CL}\left(\mathbf{R}^{d}\right)$ замкнутых непустых подмножеств $\mathbf{R}^{d}$. Через $\mathscr{E}(\mathrm{CL})$ обозначим $\sigma$-алгебру Эффроса (E. Effros) $[16]$, [17], порожденную множествами

$$
A_{V}=\{D \in \mathrm{CL}: D \cap V \neq \varnothing\}
$$

где $V$ - открытое множество $\mathbf{R}^{d}$. Тогда измеримость $F$ в смысле приведенного выше определения означает $\mathscr{H} \mid \mathscr{E}(\mathrm{CL})$-измеримость соответствуюшего однозначного отображения $F: \Omega \mapsto \mathrm{CL}$.

Обозначим через $\rho(x, y)=|x-y|$ расстояние между точками $x, y \in \mathbf{R}^{d}$ и положим

$$
\rho(x, A)=\inf _{y \in A} \rho(x, y), \quad A \subset \mathbf{R}^{d} .
$$

Топологией Вейсмана (R. Wijsman) $\tau_{W}$ на CL называется слабейшая топология, в которой все функции $\left\{\rho(x, \cdot), x \in \mathbf{R}^{d}\right\}$ непрерывны. Известно, что $\left(\mathrm{CL}, \tau_{W}\right)$ является польским пространством (т.е. сепарабельным пространством, на котором существует полная метрика, совместимая с рассматриваемой топологией) [16], [18] и борелевская $\sigma$-алгебра топологии $\tau_{W}$ совпадает с $\sigma$-алгеброй Эффроса [19], [20]. Отсюда следует, что $\mathscr{F}$-измеримое многозначное отображение $F$, рассматриваемое как случайный элемент со значениями в измеримом пространстве $(\mathrm{CL}, \mathscr{E}(\mathrm{CL}))$, обладает регулярным условным распределением относительно $\mathscr{H}[21$, гл. $2, \S 7$, п. 7].

Таким образом, сушествует функция $\mathbf{P}^{*}: \Omega \times \mathscr{E}(\mathrm{CL}) \mapsto \mathbf{R}$ со следующими свойствами:

(i) для каждого $\omega$ функция $C \mapsto \mathbf{P}^{*}(\omega, C)$ является вероятностной мерой на $\mathscr{E}(\mathrm{CL})$; 
(ii) для каждого $C \in \mathscr{E}(\mathrm{CL})$ функция $\omega \mapsto \mathbf{P}^{*}(\omega, C)$ п.н. совпадает с $\mathbf{P}(\{F \in C\} \mid \mathscr{H})(\omega)$.

На открытых множествах $V \subset \mathbf{R}^{d}$ определим регулярное условное верхнее распределение $\mu_{F}(\omega, V)=\mathbf{P}^{*}\left(\omega, A_{V}\right)$. Данная терминология (upper probabilities) соответствует работе [22], поскольку

$$
\mu_{F}(\omega, V)=\mathbf{P}(\{F \cap V \neq \varnothing\} \mid \mathscr{H})(\omega) \text { п.н. }
$$

Если $F$ - однозначное отображение, то $\mu_{F}$ - регулярное условное распределение $F$ относительно $\mathscr{H}$.

Функция $V \mapsto \mu_{F}(\omega, V)$ обладает свойством счетной субаддитивности. Действительно, пусть $V=\bigcup_{i=1}^{\infty} V_{i}$, где $V_{i}$ открыты, тогда

$$
\mu_{F}(\omega, V)=\mathbf{P}^{*}\left(\omega, A_{V}\right)=\mathbf{P}^{*}\left(\omega, \bigcup_{i=1}^{\infty} A_{V_{i}}\right) \leqslant \sum_{i=1}^{\infty} \mathbf{P}^{*}\left(\omega, A_{V_{i}}\right)=\sum_{i=1}^{\infty} \mu_{F}\left(\omega, V_{i}\right) .
$$

Носителем регулярного условного верхнего распределения отображения $F$ относительно $\mathscr{H}$ назовем множество

$$
K_{F}(\omega)=\left\{x \in \mathbf{R}^{d}: \mu_{F}\left(\omega, B_{\varepsilon}(x)\right)>0, \forall \varepsilon>0\right\} .
$$

Предложение 4. Пусть $F, F^{\prime}$ - $\mathscr{F}$-измеримье многозначные отображения с непустыми замкнутыми значениями и $F=F^{\prime}$ n.н. Тогда

(а) многозначное отображение $K_{F}$ является $\mathscr{H}$-измеримьм, замкнутьлм и $\operatorname{dom} K_{F}=\Omega$;

(b) $K_{F}=K_{F^{\prime}}$ n.н.

Д о к а 3 а т е л ь с т в о. (а) Покажем, что для любого открытого множества $V \subset \mathbf{R}^{d}$ выполняется соотношение

$$
\left\{\omega: K_{F}(\omega) \cap V \neq \varnothing\right\}=\left\{\omega: \mu_{F}(\omega, V)>0\right\}
$$

Если $K_{F}(\omega) \cap V \neq \varnothing$, то для любого $x \in K_{F}(\omega) \cap V$ найдется такое $\varepsilon>0$, что $B_{\varepsilon}(x) \subset V$ и $\mu_{F}(\omega, V) \geqslant \mu_{F}\left(\omega, B_{\varepsilon}(x)\right)>0$. Если $K_{F}(\omega) \cap V=\varnothing$, то $V$ можно представить в виде объединения открытых шаров $B_{\varepsilon(x)}(x)$ таких, что $\mu_{F}\left(\omega, B_{\varepsilon(x)}(x)\right)=0$. Пользуясь тем, что $\mathbf{R}^{d}$ обладает счетной базой, выделим из этого покрытия счетное подпокрытие $V=\bigcup_{i=1}^{\infty} B_{\varepsilon_{i}}\left(x_{i}\right), \varepsilon_{i}=\varepsilon\left(x_{i}\right)$ (см. [23, с. 25]). По свойству счетной субаддитивности отсюда следует, что $\mu_{F}(\omega, V)=0$.

Из соотношения (1.1) и $\mathscr{H}$-измеримости функции $\omega \mapsto \mu_{F}(\omega, V)$ следует, что многозначное отображение $\omega \mapsto K_{F}(\omega)$ является $\mathscr{H}$-измеримым. Кроме того, значения $K_{F}$ замкнуты: если $x \notin K_{F}(\omega)$, то для некоторого $\varepsilon>0$ имеем $\mu_{F}\left(\omega, B_{\varepsilon}(x)\right)=0$ и $B_{\varepsilon}(x) \cap K_{F}(\omega)=\varnothing$. Очевидно также, что $K_{F}(\omega) \neq \varnothing$ для всех $\omega$. 
(b) Заметим, что $\mu_{F}(\omega, V)=\mu_{F^{\prime}}(\omega, V)$ п.н. для любого открытого множества $V$. Поэтому существует такое множество $\Omega^{*}, \mathbf{P}\left(\Omega^{*}\right)=1$, что данное равенство выполнено при всех $\omega \in \Omega^{*}$ и всех $V$ из счетной базы открытых множеств пространства $\mathbf{R}^{d}$. Отсюда следует, что $K_{F}(\omega)=$ $K_{F^{\prime}}(\omega), \omega \in \Omega^{*}$. Предложение доказано.

Пусть gr $F \in \mathscr{H} \otimes \mathscr{B}$ и $\xi \in \mathscr{F} \mid \mathscr{B}$. Далее будет использоваться следующая полезная формула (см. [14, лемма 3]):

$$
\mathbf{P}(\{\xi \in F\} \mid \mathscr{H})(\omega)=\mu_{\xi}(\omega, F(\omega)) \text { п.н. }
$$

Здесь и далее $\mu_{\xi}$ - регулярное условное распределение $\xi$ относительно $\mathscr{H}$.

Известно (см. [15, с. 352]), что если $F$ - замкнутое $\mathscr{H}$-измеримое отображение и $\operatorname{dom} F=\Omega$, то любой элемент $z \in \mathscr{S}(\operatorname{conv} F, \mathscr{H})$ допускает представление

$$
z=\sum_{k=1}^{d+1} \alpha_{k} z_{k}, \quad z_{k} \in \mathscr{S}(F, \mathscr{H})
$$

где неотрицательные $\mathscr{H}$-измеримые функции $\alpha_{k}$ удовлетворяют условию $\sum_{k=1}^{d+1} \alpha_{k}=1$.

2. Формулировка основной теоремы. Если $H_{n}$ - замкнутое $\mathscr{F}_{n}$-измеримое многозначное отображение и $\mathbf{P}\left(\operatorname{dom} H_{n}\right)=1$, то через $H_{n}^{*}$ обозначим его замкнутое продолжение на $\Omega$ :

$$
H_{n}(\omega)=H_{n}^{*}(\omega), \quad \omega \in \operatorname{dom} H_{n} ; \quad H_{n}^{*}: \Omega \mapsto \mathrm{CL} .
$$

Конечно, такое продолжение неоднозначно: можно, например, положить

$$
H_{n}^{*}=H_{n} I_{\mathrm{dom} H_{n}}+\{0\} I_{\Omega \backslash \operatorname{dom} H_{n}} .
$$

Однако носители $K_{H_{n}^{*}}$ регулярных условных верхних распределений отображений $H_{n}^{*}$ относительно $\mathscr{F}_{n-1}$, с точностью до множества меры нуль, совпадают для всех таких продолжений (предложение 4(b)). Далее продолжение $H_{n}^{*}$ фиксируется произвольным образом.

Пусть теперь дана последовательность $\mathscr{F}_{n}$-измеримых открытых выпуклых многозначных отображений $\omega \mapsto G_{n}(\omega) \subset \mathbf{R}^{d}, n=0,1, \ldots, N$, с непустыми значениями. Введем последовательность $H_{n}$ следующим образом. Пусть $H_{N}=\operatorname{cl} G_{N}$. Далее, если $H_{n}$ задано и $\mathbf{P}\left(\operatorname{dom} H_{n}\right)=1$, то $H_{n-1}$ определим по формуле

$$
H_{n-1}=\operatorname{cl}\left(G_{n-1} \cap \operatorname{conv} K_{H_{n}^{*}}\right) .
$$

Согласно предложениям 3 и 4(а), графики многозначных отображений $G_{n-1} \cap \operatorname{conv} K_{H_{n}^{*}}$ принадлежат $\mathscr{F}_{n-1} \otimes \mathscr{B}$, и отображения (2.1) будут $\mathscr{F}_{n-1}$-измеримыми. 
Если же $\mathbf{P}\left(\operatorname{dom} H_{n}\right)<1$, то положим $H_{n-1}=\varnothing$. Конечно, в последнем случае окажется, что $H_{k}=\varnothing$ при всех $k<n$.

Ниже сформулирован критерий разрешимости поставленной задачи о мартингальном выборе.

Теорема 1. Следующие условия эквивалентны:

(а) существуют случайньй прочесс $S$ и эквивалентная $\mathbf{P}$ вероятностная мера $\mathrm{Q}$ такие, что $S_{n} \in \mathscr{S}\left(G_{n}, \mathscr{F}_{n}\right)$ и $S$ является Q-мартингалом;

(b) $H_{n}(\omega) \neq \varnothing$ n.н., $n=1, \ldots, N$.

При доказательстве теоремы 1 будет использован полученный в работе [3] критерий существования эквивалентной мартингальной меры для произвольного $d$-мерного случайного процесса $S$, согласованного с фильтрацией. Его можно записать следующим образом:

$$
S_{n-1}(\omega) \in \operatorname{ri}\left(\operatorname{conv} K_{S_{n}}\right)(\omega) \quad \text { п.н., } \quad n=1, \ldots, N
$$

Здесь $K_{S_{n}}$ - носитель регулярного условного распределения $S_{n}$ относительно $\mathscr{F}_{n-1}$.

Заметим, что в отличие от (2.2), где накладываются ограничения лишь на соседние элементы $S_{n-1}, S_{n}$, условие (b) нелокально. Это связано с существом дела: задача о мартингальном выборе может быть разрешима для каждой пары $G_{n}, G_{n+1}$, но неразрешима для всего процесса. Так будет, например, если $\Omega=\{\omega\}$ - одноточечное множество, $d=1, N=2, G_{0}=(0,2), G_{1}=(1,3), G_{2}=(2,4)$. Действительно, в случае одноточечного вероятностного пространства разрешимость задачи о мартингальном выборе эквивалентна условию $\bigcap_{n=0}^{N} G_{n} \neq \varnothing$.

3. Некоторые свойства носителей регулярных условных распределений. В данном пункте предполагается, что $\operatorname{dom} F=\Omega$. Как и выше, через $K_{F}$ обозначается носитель регулярного условного верхнего распределения замкнутого отображения $F$ относительно $\sigma$-алгебры $\mathscr{H} \subset \mathscr{F}$. Аналогичные обозначения используются и для носителей регулярных распределений однозначных отображений.

Лемма 1. Пусть $\left\{\xi_{i}\right\}_{i=1}^{\infty}-$ представление Кастэна для замкнутого $\mathscr{F}$-измеримого многозначного отображения $F$, тогда

$$
K_{F}(\omega)=\operatorname{cl}\left(\bigcup_{i=1}^{\infty} K_{\xi_{i}}(\omega)\right) \quad \text { n.н. }
$$

Д о к а з а т е л ь с т в о. Для любого открытого множества $V \subset \mathbf{R}^{d}$ имеем

$$
C=\{\omega: F(\omega) \cap V \neq \varnothing\}=\bigcup_{i=1}^{\infty}\left\{\omega: \xi_{i}(\omega) \in V\right\} .
$$


Пусть $C_{k}=\bigcup_{i=1}^{k}\left\{\omega: \xi_{i}(\omega) \in V\right\}$, тогда

$$
\lim _{k \rightarrow \infty} \mathbf{P}\left(C_{k} \mid \mathscr{H}\right)(\omega)=\mathbf{P}(C \mid \mathscr{H})(\omega)=\mu_{F}(\omega, V) \quad \text { п.н. }
$$

С другой стороны,

$$
\mathbf{P}\left(C_{k} \mid \mathscr{H}\right)(\omega) \leqslant \sum_{i=1}^{k} \mathbf{P}\left(\xi_{i} \in V \mid \mathscr{H}\right)(\omega)=\sum_{i=1}^{k} \mu_{\xi_{i}}(\omega, V) \quad \text { п.н. }
$$

и $\mathbf{P}\left(C_{k} \mid \mathscr{H}\right)(\omega) \geqslant \mu_{\xi_{j}}(\omega, V)$ п.н. для любого $j \leqslant k$.

Из данных соотношений следует, что на множестве $\Omega^{\prime}$ с $\mathbf{P}\left(\Omega^{\prime}\right)=1$, зависящем от $V$, верно следующее: $\mu_{F}(\omega, V)>0$, если и только если существует такой номер $i$, что $\mu_{\xi_{i}}(\omega, V)>0$. Очевидно, данное свойство можно считать выполненным на множестве $\Omega^{\prime \prime}$ с $\mathbf{P}\left(\Omega^{\prime \prime}\right)=1$ сразу для всех $V \in \mathscr{V}$, где $\mathscr{V}$ - счетная база $\mathbf{R}^{d}$.

Пусть $\omega \in \Omega^{\prime \prime}$. Если $x \notin K_{F}(\omega)$, то существует такое множество $V \in \mathscr{V}, x \in V$, что $\mu_{F}(\omega, V)=0$. При этом $\mu_{\xi_{i}}(\omega, V)=0$ для всех $i$ и

$$
V \cap \bigcup_{i=1}^{\infty} K_{\xi_{i}}(\omega)=\varnothing
$$

Следовательно, $x \notin \bigcup_{i=1}^{\infty} K_{\xi_{i}}(\omega)$ и $K_{F}(\omega) \supset \bigcup_{i=1}^{\infty} K_{\xi_{i}}(\omega)$.

Если $x \in K_{F}(\omega)$, то для любого множества $V \in \mathscr{V}$, содержащего $x$, имеем $\mu_{F}(\omega, V)>0$ и сушествует номер $i$ такой, что $\mu_{\xi_{i}}(\omega, V)>0$. Таким образом, в любой открытой окрестности точки $x$ имеются точки из $\bigcup_{i=1}^{\infty} K_{\xi_{i}}(\omega)$ и $K_{F}(\omega) \subset \operatorname{cl}\left(\bigcup_{i=1}^{\infty} K_{\xi_{i}}(\omega)\right)$. Лемма доказана.

Лемма 2. Для любого конечного или счетного набора множеств $A_{i} \in \mathscr{H}, i \in J \subset \mathbf{N}$, существует такой набор непустьх множеств $D_{i} \in \mathscr{H}, D_{i} \subset A_{i}, i \in J^{\prime} \subset J$, umo

$$
\bigcup_{i \in J} A_{i}=\bigcup_{i \in J^{\prime}} D_{i}, \quad D_{i} \cap D_{j}=\varnothing, \quad i \neq j
$$

Д о к а з а т л л с т в о. Множества

$$
D_{k}=A_{k}, \quad k=\inf J ; \quad D_{i}=A_{i} \backslash\left(\bigcup_{j \in J, j<i} A_{j}\right), \quad k<i \in J,
$$

обладают всеми требуемыми свойствами, если исключить пустые множества, т.е. положить $J^{\prime}=\left\{i \in J: D_{i} \neq \varnothing\right\}$.

Семейство $\left\{D_{i}\right\}_{i \in J^{\prime}}$ назовем разбиением, подчиненным покрытию $\left\{A_{i}\right\}_{i \in J}$ множества $A=\bigcup_{i \in J} A_{i}$.

$\mathrm{C}$ технической точки зрения доказательство следующей леммы является наиболее тонким местом данной работы. 
Лемма 3. Рассмотрим конечный набор әлементов $\xi_{i} \in \mathscr{F} \mid \mathscr{B}$, $i=0,1, \ldots, r$, и носители $K_{\xi_{i}}(\omega)$ их регулярных условных распределений относительно $\mathscr{H} \subset \mathscr{F}$. Пусть

$$
z(\omega) \in \operatorname{conv}\left(K_{\xi_{0}}(\omega) \cup \cdots \cup K_{\xi_{r}}(\omega)\right), \quad \omega \in A,
$$

где $z \in \mathscr{H} \mid \mathscr{B}, A \in \mathscr{H}$. Тогда существует такой элемент

$$
\zeta \in \mathscr{S}\left(\operatorname{conv}\left(\xi_{0}, \ldots, \xi_{r}\right), \mathscr{F}\right),
$$

umo $z(\omega) \in \operatorname{conv} K_{\zeta}(\omega)$ nри почти всех $\omega \in A$.

Д ок аз а те ль с т о. Прежде всего заметим, что достаточно рассмотреть случай $A=\Omega$. Действительно, иначе вместо $z$ рассмотрим функцию

$$
\bar{z}=z I_{A}+z^{\prime} I_{\Omega \backslash A} \in \operatorname{conv}\left(K_{\xi_{0}} \cup \cdots \cup K_{\xi_{r}}\right), \quad \omega \in \Omega,
$$

где $z^{\prime}-$ произвольный $\mathscr{H}$-измеримый селектор $\operatorname{conv}\left(K_{\xi_{0}} \cup \cdots \cup K_{\xi_{r}}\right)$.

Далее, если $\zeta=\xi$ на некотором множестве $C \in \mathscr{H}$, то $K_{\zeta}=K_{\xi}$ при почти всех $\omega \in C$. В самом деле,

$\mu_{\xi}(\omega, V) \geqslant \mathbf{P}(\{\xi \in V\} \cap C \mid \mathscr{H})=I_{C} \mathbf{P}(\{\zeta \in V\} \mid \mathscr{H})=I_{C} \mu_{\zeta}(\omega, V)$ п.н.

и, значит, $\mu_{\xi}(\omega, V) \geqslant \mu_{\zeta}(\omega, V)$ при почти всех $\omega \in C$. Противоположное неравенство доказывается аналогично.

Следовательно, для доказательства леммы достаточно найти $\mathscr{H}$-измеримое разбиение $\left\{C_{i}\right\}_{i \in L}, L \subset \mathbf{N}$, множества $\Omega$ и набор функций

$$
\zeta_{i}(\omega) \in \operatorname{conv}\left(\xi_{0}(\omega), \ldots, \xi_{r}(\omega)\right), \quad \omega \in C_{i}, \quad i \in L ; \zeta_{i} \in \mathscr{F} \mid \mathscr{B},
$$

такие, что $z \in \operatorname{conv} K_{\zeta_{i}}$ при почти всех $\omega \in C_{i}$ и всех $i \in L$. При этом функция $\zeta=\sum_{i \in L} \zeta_{i} I_{C_{i}}$ будет обладать требуемыми свойствами, поскольку $K_{\zeta}=K_{\zeta_{i}}$ при почти всех $\omega \in C_{i}$.

Воспользуемся представлением (1.3):

$$
z(\omega)=\sum_{k=1}^{d+1} \alpha_{k}(\omega) z_{k}(\omega), \quad z_{k} \in \mathscr{S}\left(K_{\xi_{0}} \cup \cdots \cup K_{\xi_{r}}, \mathscr{H}\right) .
$$

Пусть $r=1$. Каждому $\omega$ поставим в соответствие разбиение $\left\{\Psi^{s}\right\}$ множества $\{1, \ldots, d+1\}$ на классы эквивалентности: $i \sim j \Longleftrightarrow z_{i}(\omega)=$ $z_{j}(\omega)$. Множество $A^{s}$ элементов $\omega$, соответствуюших фиксированному классу эквивалентности, очевидно, принадлежит $\mathscr{H}$, а объединение всех таких множеств образует разбиение $\Omega$. На каждом множестве $A^{s}$ функция $z$ допускает представление вида (3.1), где все $z_{k}$ различны, а число 
слагаемых равно числу элементов $\Psi^{s}$. Поэтому, не ограничивая общности, будем считать, что $z_{i}(\omega) \neq z_{j}(\omega), i \neq j, i, j \in\{1, \ldots, d+1\}$.

Далее, каждой паре множеств $N^{1}$ и $N^{2}$ таких, что $N^{1} \cup N^{2}=$ $\{1, \ldots, d+1\}$ и $N^{1} \cap N^{2}=\varnothing$, поставим в соответствие множество

$$
A_{N^{1} N^{2}}=\left\{\omega \in A: \alpha_{k}(\omega) \neq 0, k \in N^{1} ; \alpha_{k}(\omega)=0, k \in N^{2}\right\} \in \mathscr{H} .
$$

Очевидно, что набор всех $A_{N^{1} N^{2}}$ образует разбиение $\Omega$, на каждом элементе которого справедливо представление вида (3.1), содержащее $\left|N^{1}\right|$ слагаемых, где все коэффициенты $\alpha_{k}$ положительны. Следовательно, без ограничения общности можно предполагать, что $\alpha_{k}(\omega)>0$ при всех $k$ и $\omega$.

Введем множества

$$
A_{k}^{i}=\left\{z_{k} \in K_{\xi_{i}}\right\}=\operatorname{dom}\left(z_{k} \cap K_{\xi_{i}}\right) \in \mathscr{H}, \quad k=1, \ldots, d+1, \quad i=0,1 .
$$

Через $\left\{D_{n}\right\}_{n \in L^{\prime}}$, где $L^{\prime} \subset\left\{1, \ldots, 2^{d+1}\right\}$, обозначим указанное в лемме 2 разбиение множества $\Omega$, подчиненное его покрытию множествами

$$
A_{n}=A_{1}^{i_{1}} \cap \cdots \cap A_{d+1}^{i_{d}+1}, \quad i_{j} \in\{0,1\}, \quad n=\sum_{k=1}^{d+1} i_{k} 2^{k-1}
$$

На каждом элементе $D_{n} \subset A_{n}$ данного разбиения положим $I_{n}=\{k$ : $\left.i_{k}=0\right\}, J_{n}=\left\{k: i_{k}=1\right\}$. Тогда

$$
z=\sum_{k \in I_{n}} \alpha_{k} x_{k}+\sum_{k \in J_{n}} \alpha_{k} y_{k}, \quad \omega \in D_{n}
$$

Здесь $x_{k}=z_{k} \in K_{\xi_{0}}, k \in I_{n} ; y_{k}=z_{k} \in K_{\xi_{1}}, k \in J_{n}$.

Рассмотрим функцию $\eta=\left(\xi_{0}, \xi_{1}\right): \Omega \mapsto \mathbf{R}^{2 d}$ и носитель $K_{\eta}(\omega)$ ее регулярного условного распределения относительно $\mathscr{H}$. Каждому множеству $D_{n}$ поставим в соответствие двудольный граф $\left(I_{n}, J_{n}, \Gamma_{n}\right)$ с множествами вершин долей $I_{n}, J_{n}$ и случайным множеством ребер $\Gamma_{n}(\omega)$, состояшим из пар $(i, j) \in I_{n} \times J_{n}$ таких, что

$$
w_{i j}(\omega)=\left(x_{i}(\omega), y_{j}(\omega)\right) \in K_{\eta}(\omega) .
$$

Пусть $\left\{U_{n}^{s}\right\}, s \in\left\{1, \ldots, m_{n}\right\}$, - семейство всех подмножеств декартова произведения $I_{n} \times J_{n}$, где $m_{n}$ - количество таких подмножеств. Рассмотрим $\mathscr{H}$-измеримое разбиение $\left\{C_{n}^{s}\right\}_{s \in L_{n}}, L_{n} \subset\left\{1, \ldots, m_{n}\right\}$, множества $D_{n}$ :

$$
\begin{aligned}
C_{n}^{s} & =\left\{\omega \in D_{n}: \Gamma_{n}(\omega)=U_{n}^{s}\right\} \\
& =\left\{\omega \in D_{n}: w_{i j} \in K_{\eta}, \quad(i, j) \in U_{n}^{s} ; w_{i j} \notin K_{\eta}, \quad(i, j) \notin U_{n}^{s}\right\} .
\end{aligned}
$$


Каждому множеству $C_{n}^{s}$ соответствует двудольный граф с фиксированным набором ребер $\Gamma_{n}=U_{n}^{s}$.

Таким образом, построено $\mathscr{H}$-измеримое разбиение $\left\{C_{n}^{s}\right\}_{s \in L_{n}}$, $n \in L^{\prime}$, множества $\Omega$, на каждом элементе $C_{n}^{s}$ которого справедливо представление (3.2), причем соответствуюший двудольный граф $\left(I_{n}, J_{n}, \Gamma_{n}\right)$ имеет фиксированные наборы вершин $I_{n}, J_{n}$ и ребер $\Gamma_{n}=U_{n}^{s}$. Следовательно, не ограничивая общности, можно считать, что данные свойства справедливы на множестве $\Omega$, и опустить все индексы, указывающие на элементы разбиения.

Итак, достаточно провести доказательство в предположении, что существует такой фиксированный двудольный граф $(I, J, \Gamma)$, что выполняются следуюшие свойства. Во-первых, элемент $z$ допускает представление

$$
\begin{gathered}
z=\sum_{k \in I} \alpha_{k} x_{k}+\sum_{k \in J} \alpha_{k} y_{k}, \quad \omega \in \Omega, \\
x_{k} \in \mathscr{S}\left(K_{\xi_{0}}, \mathscr{H}\right), \quad y_{k} \in \mathscr{S}\left(K_{\xi_{1}}, \mathscr{H}\right), \quad 0<\alpha_{k} \in \mathscr{H} \mid \mathscr{B}(\mathbf{R}) .
\end{gathered}
$$

Причем $\sum_{k \in I \cup J} \alpha_{k}=1$ и при любом $\omega \in \Omega$ все значения $x_{k}(\omega), k \in I$, $y_{k}(\omega), k \in J$, различны. Во-вторых,

$$
w_{i j} \in K_{\eta}, \quad(i, j) \in \Gamma ; \quad w_{i j} \notin K_{\eta}, \quad(i, j) \notin \Gamma, \quad \omega \in \Omega,
$$

где $\eta=\left(\xi_{0}, \xi_{1}\right), w_{i j}=\left(x_{i}, y_{j}\right)$. Заметим, что случаи $I=\varnothing$ или $J=\varnothing$ не исключаются. В любом из этих случаев $\Gamma=\varnothing$.

Покажем сначала, что сушествует такая $\mathscr{H}$-измеримая случайная величина $\delta>0$, что

$$
\begin{gathered}
B_{\delta}\left(x_{i}\right) \cap B_{\delta}\left(x_{j}\right)=\varnothing, \quad i \neq j, \\
B_{\delta}\left(y_{i}\right) \cap B_{\delta}\left(y_{j}\right)=\varnothing, \quad i \neq j, \\
\mu_{\eta}\left(\omega, B_{\delta(\omega)}\left(x_{i}(\omega)\right) \times B_{\delta(\omega)}\left(y_{j}(\omega)\right)\right)=0, \quad(i, j) \notin \Gamma,
\end{gathered}
$$

при всех $\omega \in \Omega$.

Условия (3.3), (3.4) выполняются при следующем выборе радиуса шара:

$$
\delta_{1}=\frac{1}{2} \min \left\{\min _{i \neq j}\left|x_{i}-x_{j}\right|, \min _{i \neq j}\left|y_{i}-y_{j}\right|\right\} .
$$

Пусть $\theta_{k}^{i j}(\omega)=\mu_{\eta}\left(\omega, B_{1 / k}\left(x_{i}(\omega)\right) \times B_{1 / k}\left(y_{j}(\omega)\right)\right),(i, j) \notin \Gamma$. Введем расширяющиеся с ростом $k$ последовательности множеств

$$
A_{k}^{i j}=\left\{\omega: \theta_{k}^{i j}(\omega)=0\right\} \in \mathscr{H}, \quad A_{k}^{i j} \subset A_{k+1}^{i j},
$$

и $\mathscr{H}$-измеримые функции

$$
\tau^{i j}(\omega)=I_{A_{1}^{i j}}+\sum_{k=1}^{\infty}(k+1) I_{A_{k+1}^{i j} \backslash A_{k}^{i j}}
$$


Поскольку $\left(x_{i}(\omega), y_{j}(\omega)\right) \notin K_{\eta}(\omega)$ при $(i, j) \notin \Gamma$, то $\bigcup_{k=1}^{\infty} A_{k}^{i j}=\Omega$ и для любого $\omega$ значением $\tau^{i j}(\omega)$ является наименьший номер $k$, для которого $\theta_{k}^{i j}(\omega)=0$. Следовательно, при выборе радиуса шара

$$
\delta_{2}=\min _{(i, j) \notin \Gamma}\left\{\frac{1}{\tau^{i j}}\right\}
$$

соотношения (3.5) будут выполнены. Таким образом, остается положить $\delta=\min \left\{\delta_{1}, \delta_{2}\right\}$.

Обозначим через

$\Gamma_{+}(i)=\{j \in J:(i, j) \in \Gamma\}, \quad i \in I ; \quad \Gamma_{-}(j)=\{i \in I:(i, j) \in \Gamma\}, \quad j \in J$,

множества вершин $\Gamma$, смежных заданной, и пусть $\Gamma^{1}=\left\{i \in I: \Gamma_{+}(i)=\varnothing\right\}$, $\Gamma^{2}=\left\{j \in J: \Gamma_{-}(j)=\varnothing\right\}-$ множества вершин, не имеющих смежных. Введем следующие $\mathscr{F}$-измеримые множества:

$$
\begin{aligned}
A_{i}^{\prime} & =\left\{\xi_{0} \in B_{\delta}\left(x_{i}\right)\right\}, \quad i \in \Gamma^{1} ; \quad A_{j}^{\prime \prime}=\left\{\xi_{1} \in B_{\delta}\left(y_{j}\right)\right\}, \quad j \in \Gamma^{2} ; \\
A_{i j} & =\left\{\xi_{0} \in B_{\delta}\left(x_{i}\right), \quad \xi_{1} \in B_{\delta}\left(y_{j}\right)\right\}, \quad(i, j) \in \Gamma,
\end{aligned}
$$

и $\mathscr{F}$-измеримую функцию

$$
\begin{gathered}
\zeta=\sum_{i \in \Gamma^{1}} \xi_{0} I_{A_{i}^{\prime}}+\sum_{j \in \Gamma^{2}} \xi_{1} I_{A_{j}^{\prime \prime}}+\sum_{(i, j) \in \Gamma} \zeta_{i j} I_{A_{i j}} \\
\zeta_{i j}=\left(\frac{\alpha_{i}}{\beta_{i}}+\frac{\alpha_{j}}{\gamma_{j}}\right)^{-1}\left(\frac{\alpha_{i}}{\beta_{i}} \xi_{0}+\frac{\alpha_{j}}{\gamma_{j}} \xi_{1}\right) .
\end{gathered}
$$

Здесь $\beta_{i}, \gamma_{j}$ - степени вершин $i, j$ соответственно, т.е. мощности множеств $\Gamma_{+}(i), \Gamma_{-}(j)$.

Покажем, что

$$
\begin{gathered}
x_{i} \in K_{\zeta}, \quad i \in \Gamma^{1} ; \quad y_{j} \in K_{\zeta}, \quad j \in \Gamma^{2} ; \\
z_{i j}=\left(\frac{\alpha_{i}}{\beta_{i}}+\frac{\alpha_{j}}{\gamma_{j}}\right)^{-1}\left(\frac{\alpha_{i}}{\beta_{i}} x_{i}+\frac{\alpha_{j}}{\gamma_{j}} y_{j}\right) \in K_{\zeta}, \quad(i, j) \in \Gamma,
\end{gathered}
$$

при почти всех $\omega$.

Заметим, что если $(i, j) \notin \Gamma$, то в силу соотношений $(1.2)$ и (3.5) имеем

$$
\begin{aligned}
\mathbf{P}\left(A_{i}^{\prime} \cap A_{j}^{\prime \prime} \mid \mathscr{H}\right)(\omega) & =\mathbf{P}\left(\left\{\left(\xi_{0}, \xi_{1}\right) \in B_{\delta}\left(x_{i}\right) \times B_{\delta}\left(y_{i}\right)\right\} \mid \mathscr{H}\right)(\omega) \\
& =\mu_{\eta}\left(\omega, B_{\delta(\omega)}\left(x_{i}(\omega)\right) \times B_{\delta(\omega)}\left(y_{j}(\omega)\right)\right)=0
\end{aligned}
$$

при почти всех $\omega$. Вместе с соотношениями (3.3) и (3.4) данное равенство показывает, что попарные пересечения множеств

$$
\left(A_{i}^{\prime}\right)_{i \in \Gamma^{1}}, \quad\left(A_{j}^{\prime \prime}\right)_{j \in \Gamma^{2}}, \quad\left(A_{i j}\right)_{(i, j) \in \Gamma}
$$


пусты, либо имеют нулевую меру. Отсюда следует, что

$$
\begin{gathered}
\mu_{\zeta}(\omega, V)=\mathbf{P}(\{\zeta \in V\} \mid \mathscr{H}) \\
\geqslant \max \left\{\mathbf{P}\left(\left\{\xi_{0} \in V\right\} \cap A_{i}^{\prime} \mid \mathscr{H}\right), \mathbf{P}\left(\left\{\xi_{1} \in V\right\} \cap A_{j}^{\prime \prime} \mid \mathscr{H}\right),\right. \\
\left.\quad \mathbf{P}\left(\left\{\zeta_{i j} \in V\right\} \cap A_{i j} \mid \mathscr{H}\right)\right\} \text { п.н. }
\end{gathered}
$$

для всех элементов $V$ счетной базы $\mathscr{V}$ открытых множеств $\mathbf{R}^{d}$.

Пусть $\delta_{k}=\delta / k$. При всех $k \in \mathbf{N}$ справедливы включения

$$
\begin{aligned}
&\left\{\xi_{0} \in V\right\} \cap A_{i}^{\prime} \supset\left\{B_{\delta_{k}}\left(x_{i}\right) \subset V\right\} \cap\left\{\xi_{0} \in B_{\delta_{k}}\left(x_{i}\right)\right\}, \\
&\left\{\xi_{1} \in V\right\} \cap A_{j}^{\prime \prime} \supset\left\{B_{\delta_{k}}\left(y_{j}\right) \subset V\right\} \cap\left\{\xi_{1} \in B_{\delta_{k}}\left(y_{j}\right)\right\}, \\
&\left\{\zeta_{i j} \in V\right\} \cap A_{i j} \supset\left\{B_{\delta_{k}}\left(z_{i j}\right) \subset V\right\} \cap\left\{\zeta_{i j} \in B_{\delta_{k}}\left(z_{i j}\right)\right\} \cap A_{i j} \\
& \supset\left\{B_{\delta_{k}}\left(z_{i j}\right) \subset V\right\} \cap\left\{\eta \in B_{\delta_{k}}\left(x_{i}\right) \times B_{\delta_{k}}\left(y_{j}\right)\right\} .
\end{aligned}
$$

Из предложения 3 легко вывести, что множества

$$
C_{k i}^{\prime}=\left\{B_{\delta_{k}}\left(x_{i}\right) \subset V\right\}, \quad C_{k j}^{\prime \prime}=\left\{B_{\delta_{k}}\left(y_{j}\right) \subset V\right\}, \quad C_{k i j}=\left\{B_{\delta_{k}}\left(z_{i j}\right) \subset V\right\}
$$

принадлежат $\mathscr{H}$. Поэтому, используя (1.2), находим, что при почти всех $\omega$ выполняются неравенства

$$
\begin{aligned}
& \mathbf{P}\left(\left\{\xi_{0} \in V\right\} \cap A_{i}^{\prime} \mid \mathscr{H}\right) \geqslant I_{C_{k i}^{\prime}} \mathbf{P}\left(\left\{\xi_{0} \in B_{\delta_{k}}\left(x_{i}\right)\right\} \mid \mathscr{H}\right)=I_{C_{k i}^{\prime}} \mu_{\xi_{0}}\left(\omega, B_{\delta_{k}}\left(x_{i}\right)\right), \\
& \mathbf{P}\left(\left\{\xi_{1} \in V\right\} \cap A_{j}^{\prime \prime} \mid \mathscr{H}\right) \geqslant I_{C_{k j}^{\prime \prime}} \mu_{\xi_{1}}\left(\omega, B_{\delta_{k}}\left(y_{j}\right)\right) \\
& \mathbf{P}\left(\left\{\zeta_{i j} \in V\right\} \cap A_{i j} \mid \mathscr{H}\right) \geqslant I_{C_{k i j}} \mu_{\eta}\left(\omega, B_{\delta_{k}}\left(x_{i}\right) \times B_{\delta_{k}}\left(y_{j}\right)\right) .
\end{aligned}
$$

Соотношения (3.6), (3.7) устанавливаются теперь при помощи однотипных рассуждений. Докажем, например, (3.7).

Обозначим через $\Omega^{\prime}$ множество тех $\omega$, для которых установленное выше неравенство

$$
\mu_{\zeta}(\omega, V) \geqslant I_{C_{k i j}} \mu_{\eta}\left(\omega, B_{\delta_{k}}\left(x_{i}\right) \times B_{\delta_{k}}\left(y_{j}\right)\right), \quad(i, j) \in \Gamma
$$

верно сразу для всех $V$ из счетной базы $\mathscr{V}$ и всех $k \in \mathbf{N}$. Пусть $\omega \in \Omega^{\prime}$. Для любой открытой окрестности $V \ni z_{i j}(\omega)$ выберем $k$ таким образом, чтобы $B_{\delta_{k}(\omega)}\left(z_{i j}(\omega)\right) \subset V$. При этом $\omega \in C_{k i j}$ и

$$
\mu_{\zeta}(\omega, V) \geqslant \mu_{\eta}\left(\omega, B_{\delta_{k}(\omega)}\left(x_{i}(\omega)\right) \times B_{\delta_{k}(\omega)}\left(y_{j}(\omega)\right)\right)>0
$$

так как $\left(x_{i}, y_{j}\right) \in K_{\eta},(i, j) \in \Gamma$. Это означает, что в любой открытой окрестности точки $z_{i j}(\omega) \in \mathbf{R}^{d}$ имеются точки из $K_{\zeta}(\omega)$. Следовательно, $z_{i j}(\omega) \in \operatorname{cl} K_{\zeta}(\omega)=K_{\zeta}(\omega)$. Но поскольку $\mathbf{P}\left(\Omega^{\prime}\right)=1$, то данное соотношение верно при почти всех $\omega$.

Заметим, наконец, что из определения $\beta_{i}, \gamma_{j}$ вытекает равенство

$$
\sum_{(i, j) \in \Gamma}\left(\frac{\alpha_{i}}{\beta_{i}}+\frac{\alpha_{j}}{\gamma_{j}}\right)=\sum_{i \notin \Gamma^{1}} \sum_{j \in \Gamma_{+}(i)} \frac{\alpha_{i}}{\beta_{i}}+\sum_{j \notin \Gamma^{2}} \sum_{i \in \Gamma_{-}(j)} \frac{\alpha_{j}}{\gamma_{j}}=\sum_{i \notin \Gamma^{1}} \alpha_{i}+\sum_{j \notin \Gamma^{2}} \alpha_{j} .
$$


Поэтому выражение

$$
\begin{aligned}
z & =\sum_{i \in I} \alpha_{i} x_{i}+\sum_{j \in J} \alpha_{j} y_{j} \\
& =\sum_{i \in \Gamma^{1}} \alpha_{i} x_{i}+\sum_{j \in \Gamma^{2}} \alpha_{j} y_{j}+\sum_{i \notin \Gamma^{1}} \sum_{j \in \Gamma_{+}(i)} \frac{\alpha_{i}}{\beta_{i}} x_{i}+\sum_{j \notin \Gamma^{2}} \sum_{i \in \Gamma_{-}(j)} \frac{\alpha_{j}}{\gamma_{j}} y_{j} \\
& =\sum_{i \in \Gamma^{1}} \alpha_{i} x_{i}+\sum_{j \in \Gamma^{2}} \alpha_{j} y_{j}+\sum_{(i, j) \in \Gamma}\left(\frac{\alpha_{i}}{\beta_{i}}+\frac{\alpha_{j}}{\gamma_{j}}\right) z_{i j}
\end{aligned}
$$

представляет собой выпуклую комбинацию элементов

$$
x_{i}, \quad i \in \Gamma^{1} ; \quad y_{j}, \quad j \in \Gamma^{2} ; \quad z_{i j}, \quad(i, j) \in \Gamma,
$$

и из соотношений (3.6) и (3.7) вытекает, что $z \in \operatorname{conv} K_{\zeta}$ п.н.

Итак, утверждение леммы доказано при $r=1$. Пусть оно верно при $r \leqslant m-1$ и $z \in \operatorname{conv}\left(K_{\xi_{0}} \cup \cdots \cup K_{\xi_{m}}\right)$. Из формулы (3.1) легко вывести, что элемент $z$ допускает представление

$z=\alpha x+\beta y, \quad x \in \mathscr{S}\left(\operatorname{conv}\left(K_{\xi_{0}} \cup \cdots \cup K_{\xi_{m-1}}\right), \mathscr{H}\right), \quad y \in \mathscr{S}\left(K_{\xi_{m}}, \mathscr{H}\right)$,

с $\mathscr{H}$-измеримыми коэффициентами $\alpha, \beta$ такими, что $\alpha+\beta=1, \alpha \geqslant 0$, $\beta \geqslant 0$.

По предположению индукции существует $\mathscr{F} \mid \mathscr{B}$-измеримый элемент $\zeta_{m-1} \in \operatorname{conv}\left(\xi_{0}, \ldots, \xi_{m-1}\right)$ такой, что $x \in \operatorname{conv} K_{\zeta_{m-1}}$ п.н. Следовательно,

$$
z \in \operatorname{conv}\left(\operatorname{conv} K_{\zeta_{m-1}} \cup K_{\xi_{m}}\right)=\operatorname{conv}\left(K_{\zeta_{m-1}} \cup K_{\xi_{m}}\right) \quad \text { п.н. }
$$

Еще раз применяя утверждение леммы при $r=1$, заключаем, что существует элемент

$$
\zeta \in \mathscr{S}\left(\operatorname{conv}\left(\zeta_{m-1}, \xi_{m}\right), \mathscr{F}\right) \subset \mathscr{S}\left(\operatorname{conv}\left(\xi_{0}, \ldots, \xi_{m}\right), \mathscr{F}\right)
$$

такой, что $z \in \operatorname{conv} K_{\zeta}$ п.н. Лемма 3 доказана.

Лемма 4. Пусть $F$ - замкнутое выпуклое $\mathscr{F}$-измеримое многозначное отображение и элемент $z \in \mathscr{H} \mid \mathscr{B}$ удовлетворяет условию $z \in \operatorname{conv} K_{F}$ n.н. Тогда для любой $\mathscr{H}$-измеримой функиии $\varepsilon>0$ существует $\mathscr{F} \mid \mathscr{B}$-измеримьй селектор $\zeta$ отображения ri $F$, удовлетворяющий условию $\rho\left(z, \operatorname{conv} K_{\zeta}\right)<\varepsilon n$.н.

Д о к а з а т е л ь с т в о. Покажем сначала, что для $F$ сушествует такое представление Кастэна $\left\{\eta_{i}\right\}_{i=1}^{\infty}$, что $\eta_{i} \in \operatorname{ri} F$.

Пусть $\left\{\xi_{i}\right\}_{i=1}^{\infty}$ - некоторое представление Кастэна для $F$. Из предложения 3 вытекает, что множество $A_{i}=\left\{\xi_{i} \in \operatorname{ri} F\right\}=\operatorname{dom}\left(\xi_{i} \cap \operatorname{ri} F\right)$ принадлежит F्F. Положим

$$
\eta_{i}=\xi_{i} I_{A_{i}}+\xi I_{\Omega \backslash A_{i}}
$$


где $\xi$ - произвольный $\mathscr{F} \mid \mathscr{B}$-измеримый селектор ri $F$, существующий согласно предложению 2. Функции $\eta_{i}$ являются $\mathscr{F} \mid \mathscr{B}$-измеримыми селекторами ri $F$ и

$$
\operatorname{cl}\left(\bigcup_{i=1}^{\infty} \eta_{i}\right) \supset \operatorname{cl}\left(\bigcup_{i=1}^{\infty}\left(\xi_{i} \cap \operatorname{ri} F\right)\right)=F .
$$

Справедливость последнего равенства вытекает из выпуклости $F$.

По лемме 1 имеем $z \in \operatorname{conv}\left(\operatorname{cl}\left(\bigcup_{i=1}^{\infty} K_{\eta_{i}}\right)\right)$ п.н. Изменив $z$ на множестве нулевой меры, построим $\mathscr{H} \mid \mathscr{B}$-измеримый селектор $z^{\prime}$ отображения $\operatorname{conv}\left(\operatorname{cl}\left(\bigcup_{i=1}^{\infty} K_{\eta_{i}}\right)\right)$ и воспользуемся представлением $(1.3)$ :

$$
\begin{gathered}
z^{\prime}=\sum_{k=1}^{d+1} \alpha_{k} x_{k}^{\prime}, \quad x_{k}^{\prime} \in \mathscr{S}\left(\operatorname{cl}\left(\bigcup_{i=1}^{\infty} K_{\eta_{i}}\right), \mathscr{H}\right), \\
0 \leqslant \alpha_{k} \in \mathscr{H} \mid \mathscr{B}(\mathbf{R}), \quad \sum_{k=1}^{d+1} \alpha_{k}=1 .
\end{gathered}
$$

Заменив в этом представлении $x_{k}^{\prime}$ на $\mathscr{H} \mid \mathscr{B}$-измеримые селекторы $x_{k}$ отображений $B_{\varepsilon}\left(x_{k}^{\prime}\right) \cap \bigcup_{i=1}^{\infty} K_{\eta_{i}} \neq \varnothing$, получим элемент $y=\sum_{k=1}^{d+1} \alpha_{k} x_{k}$, удовлетворяюший условию $|y-z|<\varepsilon$ п.н.

Положим $A_{k}^{i}=\left\{x_{k} \in K_{\eta_{i}}\right\} \in \mathscr{H}$ и рассмотрим покрытие множества $\Omega$, состоящее из множеств $A_{1}^{i_{1}} \cap \cdots \cap A_{d+1}^{i_{d+1}}$, где верхние индексы принимают все возможные натуральные значения. Упорядочим эти множества произвольным образом и рассмотрим $\mathscr{H}$-измеримое разбиение $\left\{D_{s}\right\}_{s=1}^{\infty}$, подчиненное данному покрытию (лемма 2). Каждому множеству $D_{s}$ соответствует набор индексов $j_{k}, k=1, \ldots, d+1$, такой, что $x_{k} \in K_{\eta_{j_{k}}}, \omega \in D_{s}$. Поэтому

$$
y \in \operatorname{conv}\left(K_{\eta_{j_{1}}}, \ldots, K_{\eta_{j_{d+1}}}\right), \quad \omega \in D_{s} .
$$

По лемме 3 сушествует такой $\mathscr{F} \mid \mathscr{B}$-измеримый элемент

$$
\zeta_{s} \in \operatorname{conv}\left(\eta_{j_{1}}, \ldots, \eta_{j_{d+1}}\right) \subset \operatorname{ri} F
$$

что $y \in \operatorname{conv} K_{\zeta_{s}}$ при почти всех $\omega \in D_{s}$. Как было отмечено в начале доказательства леммы 3 , отсюда следует, что $y \in \operatorname{conv} K_{\zeta}$ п.н., где $\zeta=$ $\sum_{s=1}^{\infty} \zeta_{s} I_{D_{s}} \in \operatorname{ri} F$. При этом

$$
\rho\left(z, \operatorname{conv} K_{\zeta}\right) \leqslant|z-y|<\varepsilon \quad \text { п.н. }
$$

Лемма 5. Пусть $\mathscr{F} \mid \mathscr{B}$-измеримье элементы $\xi, \eta$ таковьь, что $|\xi-\eta| \leqslant \varepsilon$, где $\varepsilon>0-\mathscr{H}$-измеримая функиия. Тогда для любого $z \in \mathscr{H} \mid \mathscr{B}$ виполняется неравенство

$$
\left|\rho\left(z, \operatorname{conv} K_{\xi}\right)-\rho\left(z, \operatorname{conv} K_{\eta}\right)\right| \leqslant \varepsilon \quad \text { n. } \mathcal{~} .
$$


Д о.к а з а т е л ь с т в о. Пусть $\left\{\eta_{i}\right\}_{i=1}^{\infty}$ - представление Кастэна для $\mathrm{cl}\left(\operatorname{conv} K_{\eta}\right)$. Поскольку функция

$$
h=\rho\left(z, \operatorname{conv} K_{\eta}\right)=\rho\left(z, \operatorname{cl}\left(\operatorname{conv} K_{\eta}\right)\right)=\inf _{i \in \mathbf{N}}\left|z-\eta_{i}\right|
$$

является $\mathscr{H}$-измеримой, то (согласно предложениям 2 и 3 ) существует элемент

$$
y \in \mathscr{S}\left(B_{(1+\beta) h}(z) \cap \operatorname{conv} K_{\eta}, \mathscr{H}\right) .
$$

Здесь $\beta>0$ - произвольное положительное число и $\operatorname{dom}\left(B_{(1+\beta) h}(z) \cap\right.$ conv $\left.K_{\eta}\right)=\Omega$. Данный элемент догускает представление (1.3):

$$
y=\sum_{i=1}^{d+1} \alpha_{i} y_{i}, \quad y_{i} \in \mathscr{S}\left(K_{\eta}, \mathscr{H}\right), \quad 0 \leqslant \alpha_{i} \in \mathscr{H} \mid \mathscr{B}(\mathbf{R}), \quad \sum_{i=1}^{d+1} \alpha_{i}=1 .
$$

Пусть $A_{i}=\left\{\left|\eta-y_{i}\right|<\varepsilon^{\prime}\right\}, \varepsilon^{\prime}=\beta \varepsilon$. По формуле (1.2) находим

$$
\begin{aligned}
\mathbf{P}\left(A_{i} \mid \mathscr{H}\right)(\omega) & =\mathbf{P}\left(\left\{\eta \in B_{\varepsilon^{\prime}}\left(y_{i}\right)\right\} \mid \mathscr{H}\right)(\omega) \\
& =\mu_{\eta}\left(\omega, B_{\varepsilon^{\prime}(\omega)}\left(y_{i}(\omega)\right)\right)>0 \quad \text { п.н. },
\end{aligned}
$$

поскольку $y_{i} \in K_{\eta}$. На множестве $A_{i}$ справедливо неравенство

$$
\left|\xi-y_{i}\right| \leqslant|\xi-\eta|+\left|\eta-y_{i}\right|<\varepsilon+\varepsilon^{\prime} .
$$

Следовательно,

$$
\begin{aligned}
\mu_{\xi}\left(\omega, B_{\left(\varepsilon+\varepsilon^{\prime}\right)(\omega)}\left(y_{i}(\omega)\right)\right) & =\mathbf{P}\left(\left\{\xi \in B_{\varepsilon+\varepsilon^{\prime}}\left(y_{i}\right)\right\} \mid \mathscr{H}\right)(\omega) \\
& \geqslant \mathbf{P}\left(A_{i} \mid \mathscr{H}\right)(\omega)>0 \text { п.н. }
\end{aligned}
$$

Это означает, что $B_{\varepsilon+\varepsilon^{\prime}}\left(y_{i}\right) \cap K_{\xi} \neq \varnothing$ п.н.

Рассмотрим произвольные селекторы $x_{i} \in \mathscr{S}\left(B_{\varepsilon+\varepsilon^{\prime}}\left(y_{i}\right) \cap K_{\xi}, \mathscr{H}\right)$ и положим $x=\sum_{i=1}^{d+1} \alpha_{i} x_{i} \in$ conv $K_{\xi}$ п.н. Имеет место очевидное неравенство: $|y-x|<\varepsilon+\varepsilon^{\prime}$ п.н. Таким образом,

$$
\begin{aligned}
\rho\left(z, \operatorname{conv} K_{\xi}\right) & \leqslant|z-x| \leqslant|z-y|+|y-x|<(1+\beta) h+(1+\beta) \varepsilon \\
& =\rho\left(z, \operatorname{conv} K_{\eta}\right)+\varepsilon+\beta(h+\varepsilon) \text { п.н. }
\end{aligned}
$$

Переходя к пределу при $\beta \rightarrow 0$, находим: $\rho\left(z, \operatorname{conv} K_{\xi}\right) \leqslant \rho\left(z, \operatorname{conv} K_{\eta}\right)+$ $\varepsilon$ п.н. В силу симметрии данное неравенство останется справедливым, если поменять местами $K_{\xi}$ и $K_{\eta}$. Лемма 5 доказана.

Нам также потребуется следуюший простой результат, не связанный со свойствами носителей.

Лемма 6. Пусть $F$ - открытое многозначное отображение с $\mathscr{H} \otimes \mathscr{B}$-измеримьлм графиком и $\eta \in \mathscr{S}(F, \mathscr{H})$. Тогда $\eta$ лежит в $F$ вместе с некоторой $\mathscr{H}$-измеримой трубкой, т.е. существует такая положительная $\mathscr{H}$-измеримая функиия $\theta$, что $B_{\theta}(\eta) \subset F$. 
Д о к а з а т е л ь с т в о. Пусть $\left\{\xi_{i}\right\}_{i=1}^{\infty}$ - представление Кастэна для $\mathrm{cl} F$. Положим

$$
\theta_{i}=\left|\xi_{i}-\eta\right| I_{\left\{\xi_{i} \neq \eta\right\}}+I_{\left\{\xi_{i}=\eta\right\}}>0 .
$$

Поскольку множество $\left\{\xi_{i}\right\}_{i=1}^{\infty} \backslash\{\eta\}$ плотно в $F$, то множества

$$
A_{i}=\left\{\omega: B_{\theta_{i}(\omega)}(\eta(\omega)) \subset F(\omega)\right\}
$$

образуют покрытие $\Omega$. Графики отображений $F, B_{\theta_{i}}(\eta)$ принадлежат $\mathscr{H} \otimes \mathscr{B}$. Пусть $T_{i}=\left(\mathbf{R}^{d} \backslash F\right) \cap B_{\theta_{i}}(\eta)$, тогда $A_{i}=\Omega \backslash \operatorname{dom} T_{i}$ и, следовательно, $A_{i} \in \mathscr{H}$.

Построим $\mathscr{H}$-измеримое разбиение $\left\{D_{i}\right\}_{i \in J}, J \subset \mathbf{N}$, подчиненное покрытию $\left\{A_{i}\right\}_{i=1}^{\infty}$, и введем положительную $\mathscr{H}$-измеримую функцию $\theta=\sum_{i \in J} \theta_{i} I_{D_{i}}$. Поскольку $D_{i} \subset A_{i}$, то $B_{\theta}(\eta) \subset F$.

4. Доказательство теоремы 1. Предположим, что выполнено условие (b), и выведем из него (a). Предложения 2 и 3 далее используются без специальных оговорок.

1) Прямой ход (предварительная конструкция). Положим для краткости $Y_{n}=G_{n} \cap \operatorname{conv} K_{H_{n+1}^{*}}, H_{n}=\mathrm{cl} Y_{n}, n=0,1, \ldots, N-1$. Пусть $\eta_{0}^{\prime} \in \mathscr{F}_{0} \mid \mathscr{B}$ - произвольный селектор отображения $Y_{0}$. Поскольку $Y_{0} \neq \varnothing$ п.н., элемент

$$
\eta_{0}=\eta_{0}^{\prime} I_{A_{0}}+\eta_{0}^{\prime \prime} I_{\Omega \backslash A_{0}}, \quad A_{0}=\operatorname{dom}\left(\eta_{0}^{\prime} \cap Y_{0}\right), \quad \eta_{0}^{\prime \prime} \in \mathscr{S}\left(G_{0}, \mathscr{F}_{0}\right),
$$

удовлетворяет условиям $\eta_{0} \in \operatorname{conv} K_{H_{1}^{*}}$ п.н., $\eta_{0} \in \mathscr{S}\left(G_{0}, \mathscr{F}_{0}\right)$.

В соответствии с леммой 6 выберем положительную $\mathscr{F}_{0}$-измеримую случайную величину $\theta_{0}$ таким образом, чтобы $B_{\theta_{0}}\left(\eta_{0}\right) \subset G_{0}$, и положим $\varepsilon_{0}=\theta_{0} / 2$. Согласно лемме 4 , существует такой элемент $\eta_{1} \in \mathscr{S}\left(\right.$ ri $\left.H_{1}^{*}, \mathscr{F}_{1}\right)$, что

$$
\rho\left(\eta_{0}, \operatorname{conv} K_{\eta_{1}}\right)<\varepsilon_{0} \quad \text { п.н. }
$$

Если $N=1$, то $\eta_{1} \in G_{1}$ и процесс останавливается. Если же $N \geqslant 2$, то $\eta_{1} \in$ ri $H_{1} \subset Y_{1}$ п.н. Не ограничивая общности, будем считать, что $\eta_{1} \in$ conv $K_{H_{2}^{*}}$ п.н., $\eta_{1} \in \mathscr{S}\left(G_{1}, \mathscr{F}_{1}\right)$, поскольку соотношение (4.1) не изменится, если вместо $\eta_{1}$ рассмотреть элемент

$$
\eta_{1} I_{A_{1}}+\bar{\eta}_{1} I_{\Omega \backslash A_{1}}, \quad A_{1}=\operatorname{dom}\left(\eta_{1} \cap Y_{1}\right), \quad \bar{\eta}_{1} \in \mathscr{S}\left(G_{1}, \mathscr{F}_{1}\right),
$$

отличающийся от $\eta_{1}$ лишь на множестве $\Omega \backslash A_{1}$ нулевой меры.

Пусть элементы $\eta_{j} \in \operatorname{conv} K_{H_{j+1}^{*}}$ п.н.,

$$
\begin{array}{ll}
\eta_{j} \in \mathscr{S}\left(G_{j}, \mathscr{F}_{j}\right), & j=0,1, \ldots, n-1 ; \\
\varepsilon_{j} \in \mathscr{F}_{j} \mid \mathscr{B}(\mathbf{R}), & j=0,1, \ldots, n-2,
\end{array}
$$


уже построены. Выберем $\mathscr{F}_{n-1}$-измеримую функцию $\theta_{n-1}>0$ так, чтобы выполнялось включение $B_{\theta_{n-1}}\left(\eta_{n-1}\right) \subset G_{n-1}$, и положим

$$
\varepsilon_{n-1}=\frac{1}{2} \min \left\{\varepsilon_{n-2}, \theta_{n-1}\right\} \text {. }
$$

Воспользовавшись леммой 4 , построим элемент $\eta_{n} \in \mathscr{S}\left(\right.$ гі $\left.H_{n}^{*}, \mathscr{F}_{n}\right)$, для которого справедливо неравенство

$$
\rho\left(\eta_{n-1}, \operatorname{conv} K_{\eta_{n}}\right)<\varepsilon_{n-1} \quad \text { п.н. }
$$

Как и выше, не ограничивая общности, мы можем считать, что $\eta_{n} \in \operatorname{conv} K_{H_{n+1}^{*}}$ п.н., $\eta_{n} \in \mathscr{S}\left(G_{n}, \mathscr{F}_{n}\right)$, так как неравенство (4.2) сохраняется при замене $\eta_{n}$ на элемент

$$
\eta_{n} I_{A_{n}}+\bar{\eta}_{n} I_{\Omega \backslash A_{n}}, \quad A_{n}=\operatorname{dom}\left(\eta_{n} \cap Y_{n}\right), \quad \bar{\eta}_{n} \in \mathscr{S}\left(G_{n}, \mathscr{F}_{n}\right),
$$

обладающий такими свойствами.

Таким образом, построен согласованный с фильтрацией случайный процесс $\left(\eta_{n}\right)_{n=0}^{N}, \eta_{n} \in G_{n}$, удовлетворяющий условиям (4.2) при всех $n=1, \ldots, N$.

2) Обратный ход (деформация). Введем следующую последовательность случайных величин:

$$
\delta_{N-1}=\varepsilon_{N-1}, \quad \delta_{k}=2 \varepsilon_{k}, \quad k \leqslant N-2 .
$$

Заметим, что $B_{\delta_{k}}\left(\eta_{k}\right) \subset G_{k}$, так как $\delta_{k} \leqslant \theta_{k}$.

Положим $\xi_{N}=\eta_{N}$ и построим $\mathscr{F}_{N-1} \mid \mathscr{B}$-измеримый элемент

$$
\xi_{N-1} \in B_{\delta_{N-1}}\left(\eta_{N-1}\right) \cap \operatorname{ri}\left(\operatorname{conv} K_{\xi_{N}}\right) .
$$

При этом $\xi_{N-1} \in G_{N-1}$ п.н., так как $B_{\delta_{N-1}}\left(\eta_{N-1}\right) \subset G_{N-1}$ и множество в правой части последнего соотношения непусто при почти всех $\omega$ в силу (4.2).

Предположим, что элементы

$$
\xi_{j} \in \mathscr{S}\left(B_{\delta_{j}}\left(\eta_{j}\right) \cap \operatorname{ri}\left(\operatorname{conv} K_{\xi_{j+1}}\right), \mathscr{F}_{j}\right), \quad j=n, \ldots, N-1,
$$

уже построень. Поскольку $\left|\xi_{n}-\eta_{n}\right|<\delta_{n}=2 \varepsilon_{n} \leqslant \varepsilon_{n-1}$, то, согласно лемме 5 и неравенству (4.2), имеем

$$
\rho\left(\eta_{n-1}, \operatorname{conv} K_{\xi_{n}}\right) \leqslant \rho\left(\eta_{n-1}, \operatorname{conv} K_{\eta_{n}}\right)+\varepsilon_{n-1}<2 \varepsilon_{n-1}=\delta_{n-1} \quad \text { п.н. }
$$

Оंтсюда следует, что $B_{\delta_{n-1}}\left(\eta_{n-1}\right) \cap \operatorname{ri}\left(\operatorname{conv} K_{\xi_{n}}\right) \neq \varnothing$ п.н. и любой $\mathscr{F}_{n-1} \mid \mathscr{B}$-измеримый селектор

$$
\xi_{n-1} \in B_{\delta_{n-1}}\left(\eta_{n-1}\right) \bigcap \operatorname{ri}\left(\operatorname{conv} K_{\xi_{n}}\right)
$$

удовлетворяет условию $\xi_{n-1} \in B_{\delta_{n-1}}\left(\eta_{n-1}\right) \subset G_{n-1}$ п.н. Таким образом, построен согласованный с фильтрацией случайный процесс $\left(\xi_{n}\right)_{n=0}^{N}$, $\xi_{n} \in G_{n}$ п.н., обладающий следуюшим свойством:

$$
\xi_{n-1} \in \operatorname{ri}\left(\operatorname{conv} K_{\xi_{n}}\right) \quad \text { п.н., } \quad n=1, \ldots, N \text {. }
$$


Пусть $C_{n}=\left\{\xi_{n} \in G_{n}\right\}$ и $\zeta_{n} \in \mathscr{F}_{n} \mid \mathscr{B}-$ произвольный селектор $G_{n}$. Положим

$$
S_{n}=\xi_{n} I_{C_{n}}+\zeta_{n} I_{\Omega \backslash C_{n}} \in \mathscr{S}\left(G_{n}, \mathscr{F}_{n}\right) .
$$

Поскольку $\mathbf{P}\left(C_{n}\right)=1$, то $K_{S_{n}}=K_{\xi_{n}}$ п.н. и выполняется соотношение (2.2). Следовательно, для процесса $S$ существует эквивалентная мартингальная мера.

Доказательство импликации $(\mathrm{a}) \Longrightarrow(\mathrm{b})$ гораздо проще. Пусть $S$ случайный процесс, указанный в условии (а). В силу (2.2) справедливо соотношение $S_{n-1} \in G_{n-1} \cap \operatorname{ri}\left(\operatorname{conv} K_{S_{n}}\right)$ п.н., $n=1, \ldots, N$. Заметим, что $S_{N} \in H_{N}=\operatorname{cl} G_{N}$. Пусть уже установлено, что $S_{j} \in H_{j}$ п.н., $j=n, \ldots, N$. Очевидно, что $K_{S_{n}} \subset K_{H_{n}}$ п.н., поэтому

$$
S_{n-1} \in G_{n-1} \cap \operatorname{ri}\left(\operatorname{conv} K_{S_{n}}\right) \subset G_{n-1} \cap \operatorname{conv} K_{H_{n}} \subset H_{n-1} \quad \text { п.н. }
$$

В частности, $H_{n} \neq \varnothing$ п.н. при всех $n$. Теорема доказана.

5. Случай конечного вероятностного пространства. Предположение о конечности вероятностного пространства позволяет дать элементарное доказательство основной теоремы и несколько ее усилить. Итак, пусть множество $\Omega$ конечно, а вероятностная мера $\mathbf{P}$ невырождена: $\mathbf{P}(\omega)>0$. Ясно, что в рассматриваемом случае нет различия между алгебрами и $\sigma$-алгебрами, а также между условиями «всюду» и «почти всюду».

Пусть алгебра $\mathscr{H} \subset \mathscr{F}$ порождается разбиением $\left\{D_{1}, \ldots, D_{l}\right\}$. Его элементы называются атомами $\mathscr{H}$. Многозначное отображение является $\mathscr{H}$-измеримым, если оно постоянно на атомах $\mathscr{H}$. Условное верхнее распределение $\mathscr{F}$-измеримого многозначного отображения $F$ с непустыми замкнутыми значениями относительно алгебры $\mathscr{H}$ определяется следующей формулой:

$$
\mu_{F}(\omega, V)=\mathbf{P}(\{F \cap V\} \mid \mathscr{H})=\sum_{i=1}^{l} \frac{\mathbf{P}\left(\left\{\omega^{\prime} \in D_{i}: F\left(\omega^{\prime}\right) \cap V \neq \varnothing\right\}\right)}{\mathbf{P}\left(D_{i}\right)} I_{D_{i}}(\omega) .
$$

Носитель $\mu_{F}$ имеет вид

$$
K_{F}(\omega)=\bigcup_{\omega^{\prime} \in D_{i}} F\left(\omega^{\prime}\right), \quad \omega \in D_{i}, \quad i=1, \ldots, l .
$$

Рассмотрим фильтрацию $\left(\mathscr{F}_{n}\right)_{n=0}^{N}$ и последовательность $\mathscr{F}_{n}$-измеримых выпуклых многозначных отображений $\omega \mapsto G_{n}(\omega) \subset \mathbf{R}^{d}$ с непустыми значениями (условие открытости $G_{n}$ здесь не накладывается). Пусть $W_{N}=\operatorname{cl} G_{N}$. Если отображение $W_{n}$ определено и $\operatorname{dom} W_{n}=\Omega$, то положим

$$
W_{n-1}=\operatorname{cl}\left(\left(\operatorname{ri} G_{n-1}\right) \cap \operatorname{ri}\left(\operatorname{conv} K_{W_{n}}\right)\right) .
$$

Здесь $K_{W_{n}}$ - носитель регулярного условного верхнего распределения $W_{n}$ относительно $\mathscr{F}_{n-1}$. Если же $\mathbf{P}\left(\operatorname{dom} W_{n}\right)<1$, то будем считать, что $W_{n-1}=\varnothing$. 
Теорема 2. Пусть множество $\Omega$ конечно и мера $\mathbf{P}$ невырождена, тогда следующие условия эквивалентнь:

(а) существуют случайньй прочесс $S$ и невьгожденная вероятностная мера $\mathrm{Q}$ такие, ито $S_{n} \in \mathscr{S}\left(\right.$ ri $\left.G_{n}, \mathscr{F}_{n}\right)$ u $S$ является Q-мартингалом;

(b) $W_{n}(\omega) \neq \varnothing$ при всех $\omega$ un.

Д ок а з а тель с т в о. Пусть выполнено условие (b). В качестве $S_{0}$ возьмем произвольный $\mathscr{F}_{0}$-измеримый селектор отображения ri $W_{0}$. Пусть элементы $S_{j} \in \mathscr{S}\left(\operatorname{ri} W_{j}, \mathscr{F}_{j}\right), j=0,1, \ldots, n-1$, уже построены. Предположим, что $\omega \in D_{i}$, где $D_{i}$ - атом алгебры $\mathscr{F}_{n-1}$. Используя известное представление относительной внутренности объединения (см. [24, теорема 6.9]), находим

$$
\begin{aligned}
& S_{n-1}(\omega) \in \operatorname{ri}\left(\operatorname{conv} K_{W_{n}}\right)(\omega)=\operatorname{ri}\left(\operatorname{conv}\left(\bigcup_{\omega^{\prime} \in D_{i}} W_{n}\left(\omega^{\prime}\right)\right)\right) \\
& \quad=\left\{\sum_{\omega^{\prime} \in D_{i}} \lambda_{i}\left(\omega^{\prime}\right) x_{i}\left(\omega^{\prime}\right): x_{i}\left(\omega^{\prime}\right) \in \operatorname{ri} W_{n}\left(\omega^{\prime}\right), \lambda_{i}\left(\omega^{\prime}\right)>0, \sum_{\omega^{\prime} \in D_{i}} \lambda_{i}\left(\omega^{\prime}\right)=1\right\} .
\end{aligned}
$$

Здесь и далее имеется в виду, что $x_{i}$ и $\lambda_{i}\left(\right.$ как и $\left.W_{n}\right)$ постоянны на атомах алгебры $\mathscr{F}_{n}$, содержащихся в $D_{i}$.

Итак, $S_{n-1}=\sum_{\omega^{\prime} \in D} \lambda_{i}\left(\omega^{\prime}\right) x_{i}\left(\omega^{\prime}\right)$ на атоме $D_{i}$ при некоторых $x_{i} \in \mathrm{ri} W_{n}$ и $\lambda_{i}>0$. Положим $S_{n}(\omega)=x_{i}(\omega), \omega \in D_{i}$. Очевидно, что $S_{n} \in \mathscr{S}\left(\operatorname{ri} W_{n}, \mathscr{F}_{n}\right)$ и на каждом атоме $D_{i}$ выполняется соотношение

$$
\begin{aligned}
S_{n-1}(\omega) & =\sum_{\omega^{\prime} \in D_{i}} \lambda_{i}\left(\omega^{\prime}\right) S_{n}\left(\omega^{\prime}\right) \in \operatorname{ri}\left(\operatorname{conv}\left(\bigcup_{\omega^{\prime} \in D_{i}}\left\{S_{n}\left(\omega^{\prime}\right)\right\}\right)\right) \\
& =\operatorname{ri}\left(\operatorname{conv} K_{S_{n}}\right)(\omega) .
\end{aligned}
$$

Согласно критерию (2.2), отсюда вытекает условие (а).

Докажем импликацию $(\mathrm{a}) \Longrightarrow(\mathrm{b})$. В силу условия (а) и критерия (2.2) имеем $S_{n-1} \in \operatorname{ri} G_{n-1} \cap \operatorname{ri}\left(\operatorname{conv} K_{S_{n}}\right), \quad n=1, \ldots, N$. Очевидно, что $S_{N} \in \operatorname{ri} W_{N}$. Пусть $S_{j} \in \operatorname{ri} W_{j}, j=n, \ldots, N$. Поскольку $K_{S_{n}} \subset K_{W_{n}}$, то

$$
S_{n-1} \in \operatorname{ri} G_{n-1} \bigcap \operatorname{ri}\left(\operatorname{conv} K_{W_{n}}\right)=\operatorname{ri} W_{n-1} \neq \varnothing .
$$

Теорема 2 доказана.

Основное отличие данного результата от общего случая состоит в отсутствии требования открытости $G_{n}$. Представляет интерес исследование вопроса о возможности подобного обобщения теоремы 1.

Заметим, что в определении $W_{n}$ и условии теоремы 2 нельзя заменить $\mathrm{ri} G_{n}$ на $G_{n}$, даже если множества $G_{n}$ замкнуты. Действительно, пусть $\Omega=\{\omega\}$ - одноточечное множество, $d=N=1, G_{0}=[0,1]$, $G_{1}=[1,2]$. Тогда набор $S_{i}=1 \in G_{i}, i=0,1$, образует мартингал относительно единственной вероятностной меры на $\Omega$, но $G_{0} \cap \operatorname{ri}\left(\operatorname{conv} K_{G_{1}}\right)=$ $G_{0} \cap \operatorname{ri} G_{1}=\varnothing$. 


\section{СПИСОК ЛИТЕРАТУРЫ}

1. Harrison J.M., Pliska S.R. Martingales and stochastic integrals in the theory of continuous trading. - Stochastic Process. Appl., 1981, v. 11, № 3, p. 215-260.

2. Dalang R.C., Morton A., Willinger W. Equivalent martingale measures and noarbitrage in stochastic securities market models. - Stochastics Stochastics Rep., 1990, v. 29 , № 2, p. 185-201.

3. Jacod J., Shiryaev A.N. Local martingales and the fundamental asset pricing theorems in the discrete-time case. - Finance Stoch., 1998, v. 2, № 3, p. 259-273.

4. Ширяев A.H. Основы стохастической финансовой математики. Т. 2. Теория. М.: Фазис, 1998, 528 c.

5. Jouini E., Kallal H. Martingales and arbitrage in securities markets with transaction costs. - J. Econom. Theory, 1995, v. 66, № 1, p. 178-197.

6. Kabanov $Y$., Stricker $C$. The Harrison-Pliska arbitrage pricing theorem under transaction costs. - J. Math. Econom., 2001, v. 35, № 2, p. 185-196.

7. Kabanov Y., Rásonyi M., Stricker C. No-arbitrage criteria for financial markets with efficient friction. - Finance Stoch., 2002, v. 6, № 3, p. 371-382.

8. Schachermayer $W$. The fundamental theorem of asset pricing under proportional transaction costs in finite discrete time. - Math. Finance, 2004, v. 14, № 1, p. 19-48.

9. Булинский $A . B$. ШШряев $A . H$. Теория случайных процессов. М.: Физматлит, 2003, $400 \mathrm{c}$.

10. Himmelberg C. J. Measurable relations. -- Fund. Math., 1975, v. 87, p. 53-72.

11. Левин В.Л. Выпуклый анализ в пространствах измеримых функций и его применения в математике и экономике. М.: Наука, 1985, 352 с.

12. Евстигнеев И. В. Теоремы измеримого выбора и вероятностные модели управления в общих топологических пространствах. - Матем. сб., 1986, т. 131, № 1, с. $27-39$.

13. Богачев В.И. Основы теории меры. Т. 2. М.-Ижевск: НИЦ «Регулярная и хаотическая динамика», 2003, 576 с.

14. Рохлин Д. Б. Расширенная версия теоремы Даланга-Мортона-Виллинджера при выпуклых ограничениях на портфель. - Теория вероятн. и ее примен., 2004, т. 49 , в. 3 , с. $503-521$.

15. Иоффе А.Д., Тихомиров В.М. Теория экстремальных задач. М.: Наука, 1974, $479 \mathrm{c}$.

16. Beer G. A Polish topology for the closed subsets of a Polish space. - Proc. Amer. Math. Soc., 1991, v. 113, № 4, p. 1123-1133.

17. Barbati A., Beer G., Hess C. The Hausdorff metric topology, the Attouch-Wets topology, and the measurability of set-valued functions. - J. Convex Anal., 1994, v. 1, № 1, p. 107-119.

18. Zsilinszky L. Polishness of the Wijsman topology revisited. - Proc. Amer. Math. Soc., 1998, v. 126, № 12, p. 3763-3765.

19. Hess C. Loi de probabilité des ensembles aléatoires à valeurs fermées dans un espace métrique séparable. - C. R. Acad. Sci. Paris, 1983, v. 296, № 21, p. 883-886.

20. Hess C. Contributions à l'étude de la mesurabilité, de la loi de probabilité, et de la convergence des multifunctions. - Thèse d'état. Montpellier: Université Montpellier II, 1986.

21. Ширяев А.Н. Вероятность. М.: Наука, 1980, 576 с.

22. Dempster A.P. Upper and lower probabilities induced by a multivalued mapping. Ann. Math. Statist., 1967, v. 38, № 2, p. 325-339.

23. Александрян Р. А., Мирзаханян Э. А. Обшая топология. М.: Высшая школа, 1979, $336 \mathrm{c}$.

24. Рокафеллар Р. Т. Выпуклый анализ. М.: Мир, 1973, 469 с.

Поступила в редакцию

2.VIII. 2004

Исправјенный вариант

14.III. 2005 